С. А. Голубков ${ }^{1}$, Ю. Б. Гуров ${ }^{2}$, К. Н. Гусев, Н. Н. Егоров ${ }^{1}$, Н. И. Замятин, С. Л. Катулина, Ю. Ф. Козлов ${ }^{1}$, К. А. Коньков ${ }^{1}$, В. Г. Сандуковский, А. И. Сидоров ${ }^{1}$, А. С. Старостин ${ }^{3}$

ИССЛЕДОВАНИЕ ЭФФЕКТА ВНУТРЕННЕГО УСИЛЕНИЯ НА ПЛАНАРНЫХ $p^{+} n n^{+}$-СТРУКТУРАХ ИЗ ВЫСОКООМНОГО КРЕМНИЯ

Направлено в журнал «Приборы и техника эксперимента»

${ }^{1}$ Научно-исследовательский институт материаловедения, Зеленоград, Россия

${ }^{2}$ Московский инженерно-физический институт, Москва

${ }^{3}$ Институт теоретической и экспериментальной физики, Москва 
Голубков С. А. и др.

P13-2004-26

Исследование эффекта внутреннего усиления на планарных

$p^{+} n n^{+}$-структурах из высокоомного кремния

Представлены первые результаты исследований специальных стриповых и пиксельных детекторов из кремния, структуры которых позволяют создавать в области $p$ - $n$-перехода электрическое поле $\sim 5 \cdot 10^{5} \mathrm{~B} / \mathrm{cm}$, достаточное для лавинного размножения носителей заряда. Показана возможность реализации в полупроводниковом детекторе режима внутреннего усиления, сходного с пропорциональным усилением в газовых счетчиках. Спектр альфа-частиц ${ }^{238} \mathrm{Pu}$ $\left(E_{\alpha}=5,5 \mathrm{MэB}\right)$ демонстрирует «усиленный» пик со значениями энергии 70,2 МэВ и энергетическим разрешением FWHM = 10,2 МэВ.

Работа выполнена в Лаборатории ядерных проблем им. В. П. Джелепова ОИЯИ.

Препринт Объединенного института ядерных исследований. Дубна, 2004

Перевод авторов

Golubkov S. A. et al.

Investigation of Internal Amplification Effect at Planar

$\left(p^{+} n n^{+}\right)$Structures Made of High Resistivity Silicon

The first results of investigations of special strip and pixel silicon detectors are presented. The detector structures allow creating the high electric field (about $5 \cdot 10^{5} \mathrm{~V} / \mathrm{cm}$ ) near $p-n$ junction. This field is high enough for avalanche multiplication of charge carriers. The possibility of internal amplification in the semiconductor detector similar to proportional amplification in gaseous counters is shown. The spectrum of $\alpha$-particles of the ${ }^{238} \mathrm{Pu}\left(E_{\alpha}=5.5 \mathrm{MeV}\right)$ demonstrates the «amplifying» peak at the energy of $70.2 \mathrm{MeV}$ and energy resolution $\mathrm{FWHM}=10.2 \mathrm{MeV}$.

The investigation has been performed at the Dzhelepov Laboratory of Nuclear Problems, JINR. 
Для решения ряда проблем современной неускорительной физики требуются спектрометры с очень низким порогом регистрации излучения (ниже одного килоэлектронвольта), высоким энергетическим разрешением (FWHM $\sim 3-5 \%$ ) и максимально возможной эффективностью регистрации (большим чувствительным объемом детектора). Примерами таких задач являются проводимые и планируемые эксперименты по поиску темной материи [1], измерению магнитного момента нейтрино [2] и когерентного рассеяния нейтрино на ядрах [3]. Используемые сейчас для этих целей установки на основе криогенных детекторов-болометров [4] имеют сложную конструкцию и высокую стоимость. При этом активный объем данных детекторов не превышает пока 20-30 $\mathrm{cm}^{3}$. Стандартные полупроводниковые детекторы (ППД) из германия обладают высоким энергетическим разрешением (2,5-3\% в области энергии 2-6 кэВ) и позволяют создавать спектрометры с чувствительным объемом до $500 \mathrm{~cm}^{3}$. Однако они имеют сравнительно высокий энергетический порог ( $\sim 2$ кэВ), который определяется соотношением наведенного частицей заряда и шумов токов утечки детектора. Можно понизить энергетический порог, если создать в детекторе режим лавинного размножения (мультипликации) зарядов, т.е. внутреннего усиления сигналов. Это позволило бы выделить сигнал низкой энергии из шумов, что невозможно в стандартном ППД.

Обычно приборы с внутренним усилением, например лавинные фотодиоды (ЛФД), разрабатываются для регистрации света. Как правило, это приборы с небольшой площадью $\left(10-50\right.$ мм $\left.^{2}\right)$ и толщиной чувствительной области 10-50 мкм [5,6]. Площадь ЛФД ограничена принципиальными технологическими проблемами: однородностью исходного материала, однородностью легирования и др., а большая толщина активной области для регистрации видимого света не требуется. С другой стороны, с увеличением площади ЛФД возрастает емкость для «тонких» структур. Поэтому для задач, где необходима минимальная емкость ЛФД, их изготавливают на высокоомном кремнии или выращивают «толстые» (более 100 мкм) эпитаксиальные высокоомные слои.

В работе [7] опубликованы результаты разработки ЛФД большой площади $\left(10 \mathrm{~cm}^{2}\right)$. Авторы изготовили ЛФД на основе нейтронно-легированного кремния $n$-типа проводимости методом глубокой диффузии галлия. Такой прибор стабильно работает с коэффициентом усиления 1000 при напряжении 200 B. 
Известно еще несколько типов ППД с внутренним усилением:

- приборы, в которых для реализации усиления применялся принцип работы транзистора [8];

- кремниевые DEPFET-матрицы [9], разработанные для экспериментов в физике высоких энергий, которые совмещают в себе полевой транзистор (FET) с дрейфовой камерой;

— детекторы на основе гетеропереходов между материалами с различной шириной запрещенных зон [10], в которых электроны получают энергию, необходимую для вторичной ионизации, при переходе в материал с меньшей шириной запрещенной зоны;

- кремниевые лавинные приборы с отрицательной обратной связью (ЛОС-диоды) на основе структур МРП (металл — резистивный слой — полупроводник) [11].

Каждый из вышеназванных типов детекторов с внутренним усилением обладает характерными достоинствами и недостатками. Но главный общий недостаток - маленький чувствительный объем детекторов. При этом перечисленные методы получения режимов внутреннего усиления не позволяют надеяться на создание требуемых чувствительных объемов для задач [1-3].

Для достижения ультранизкого энергетического порога в детекторе большого объема из сверхчистого германия (HPGe) был предложен метод внутреннего пропорционального усиления заряда [12]. Согласно расчетам такой подход предполагает получение энергетического порога $\sim 10$ эВ в чувствительном объеме $\sim 100 \mathrm{~cm}^{3}$.

На первом этапе для исследования принципа внутреннего усиления в ППД, сходного с пропорциональным усилением в газовых счетчиках, было решено использовать кремниевые детекторы, изготовленные по планарной технологии (SiD), а затем перейти к разработке HPGe-прототипа. В основе этого решения лежат следующие соображения: для $\mathrm{SiD}$ развита надежная технология изготовления микроструктур и имеется отечественная производственная база; создание $\mathrm{SiD}$ относительно недорого, что позволяет проверить целый ряд конструктивных и технологических решений.

Планарная технология, развитая в НИИМВ (г. Зеленоград) для ускорительных экспериментов с высокими радиационными потоками [13], позволяет изготавливать детекторы из кремния $n$-типа с напряженностью пробоя свыше $3 \cdot 10^{4} \mathrm{~B} / \mathrm{cm}$. Недостатком использования $n$-кремния для внутреннего усиления является то, что в область возможной мультипликации заряда будут попадать только дырки, подвижность которых при комнатной температуре в 3 раза меньше подвижности электронов. Поэтому для реализации режима внутреннего усиления потребуется более высокая напряженность поля, чем в детекторе из кремния $p$-типа. Понимая проигрыш в усилении для дырок на $n$-кремнии, мы сознательно начали исследования на этом типе материала, чтобы экспериментально проверить на современном технологическом уровне 
возможность создания реальных опытных структур с эффектом усиления. Кроме того, подвижность дырок значительно увеличивается при охлаждении кремния до температуры жидкого азота $\left(10^{4} \mathrm{~cm}^{2} /(\mathrm{B} \cdot \mathrm{c})\right)$ [14], что делает проблему усиления дырок менее принципиальной. Очевидно, что, создавая методом селективного травления с последующей имплантацией профиль $p^{+} n$-перехода в виде пирамиды, можно заметно повысить напряженность электрического поля в области острия пирамиды.

В настоящей работе представлены первые результаты исследований специальных стриповых и пиксельных детекторов из кремния, структуры которых позволяют создавать в области $p$ - $n$-перехода электрическое поле $\sim 5 \cdot 10^{5} \mathrm{~B} / \mathrm{cm}$, достаточное для лавинного размножения носителей заряда. Детекторы были разработаны и изготовлены по планарной технологии в НИИМВ (г. Зеленоград). Исходным материалом служили пластины кремния $n$-типа толщиной 300 мкм с удельным сопротивлением 3 кОм · см и кристаллографической ориентацией $\langle 100\rangle$ по нормали к поверхности.

\section{СТРИПОВЫЕ ДЕТЕКТОРЫ}

Структура детекторов представлена на рис. 1 и 2. На пластине кремния размещены четыре квадранта, в каждом из которых ионной имплантацией бора создано по три полоски, называемых стрипами, шириной 150 мкм и длиной 7,5 мм. Межстриповое расстояние составляет 2,8 мм. Для повышения напряжения электрического пробоя по поверхности планарной структуры создаются охранные $p^{+}$-кольца (см. рис.1). Крайние стрипы в квадранте имеют клиновидную (пирамидальную) форму (см. рис. 2) с различной шириной основания клина - 6 и 15 мкм. Средний стрип (тестовый) имел стандартный профиль и был предназначен для сравнительных измерений. Обратная сторона пластины являлась омическим контактом, общим для всех детекторов. Режимы имплантации были следующими: энергия внедрения ионов бора ( $p^{+}$-контакт) $E_{\mathrm{B}}=50$ кэВ, доза $D_{\mathrm{B}}=100 \div 200$ мкКл/см ${ }^{2}$; энергия внедрения ионов фосфора ( $n^{+}$-контакт) $E_{\mathrm{P}}=100$ кэВ, доза $D_{P}=200 \div 300$ мкКл/см ${ }^{2}$. Токи утечки детекторов при комнатной температуре при значении напряжения смещения 500 В не превышали 3 нА.

Для исследования режима внутреннего усиления использовались альфачастицы от источников ${ }^{238} \mathrm{Pu}$ и ${ }^{226} \mathrm{Ra}$. Облучение производилось со стороны $p$ - $n$-перехода и со стороны омического контакта. Были проведены серии измерений при комнатной температуре $(300 \mathrm{~K})$ и при температуре жидкого азота $(77 \mathrm{~K})$.

Типичные спектры от источника ${ }^{226} \mathrm{Ra}$ для различных напряжений, приложенных к детектору, представлены на рис. 3. Наличие пиков в начальных каналах при облучении со стороны $p$ - $n$-перехода (рис. $3, a, \sigma)$ обусловлено 


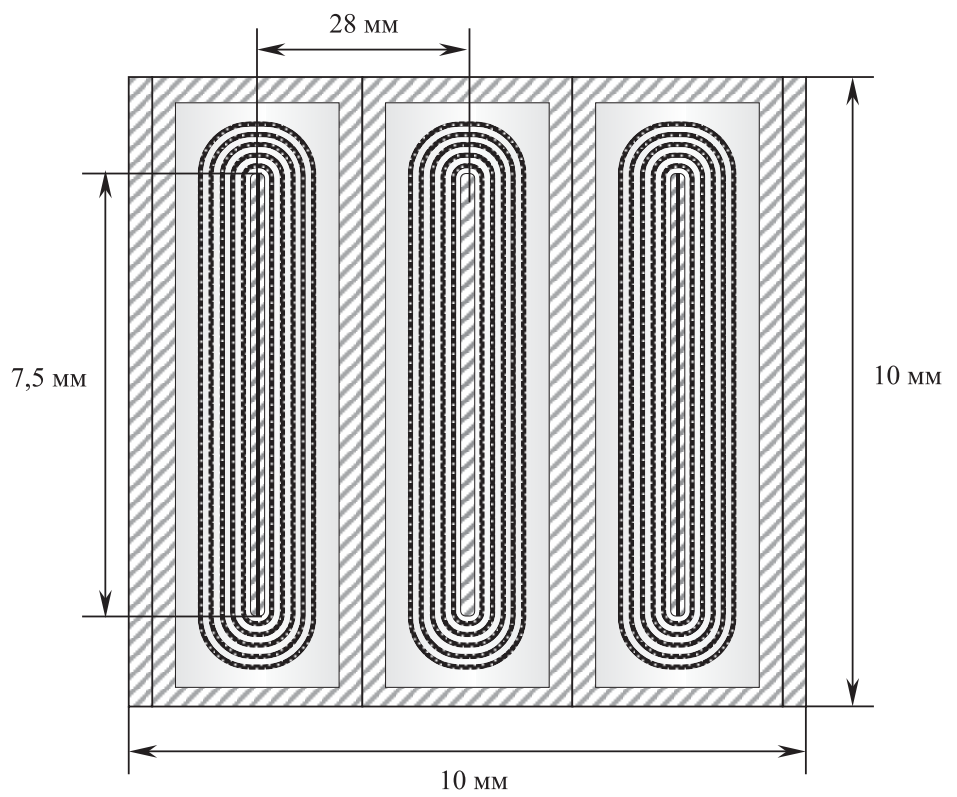

Рис. 1. Структура поверхности стриповых детекторов

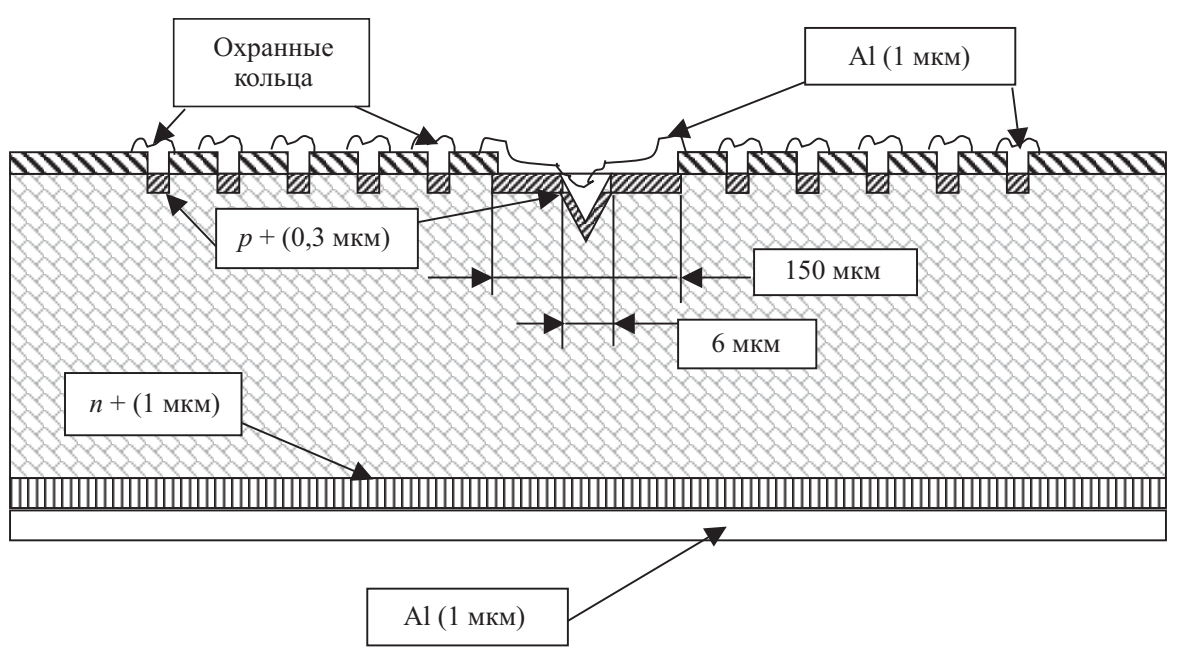

Рис. 2. Структура стрипового детектора с клиновидным $p$ - $n$-переходом 


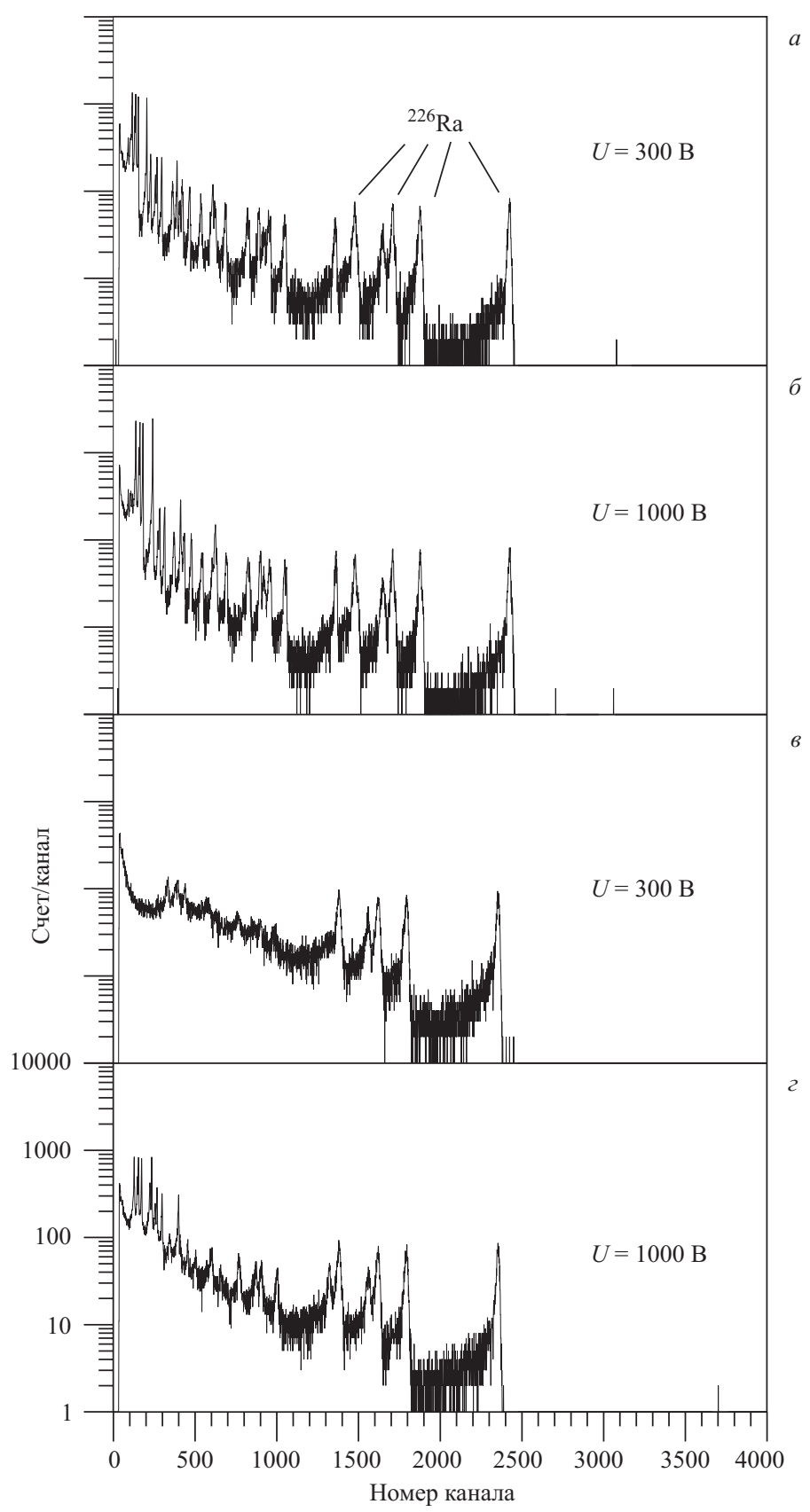

Рис. 3. Спектры альфа-частиц, полученные с помощью стрипового детектора при облучении со стороны $p$ - $n$-перехода $(a, \sigma)$ и омического контакта $(6,2)$ 


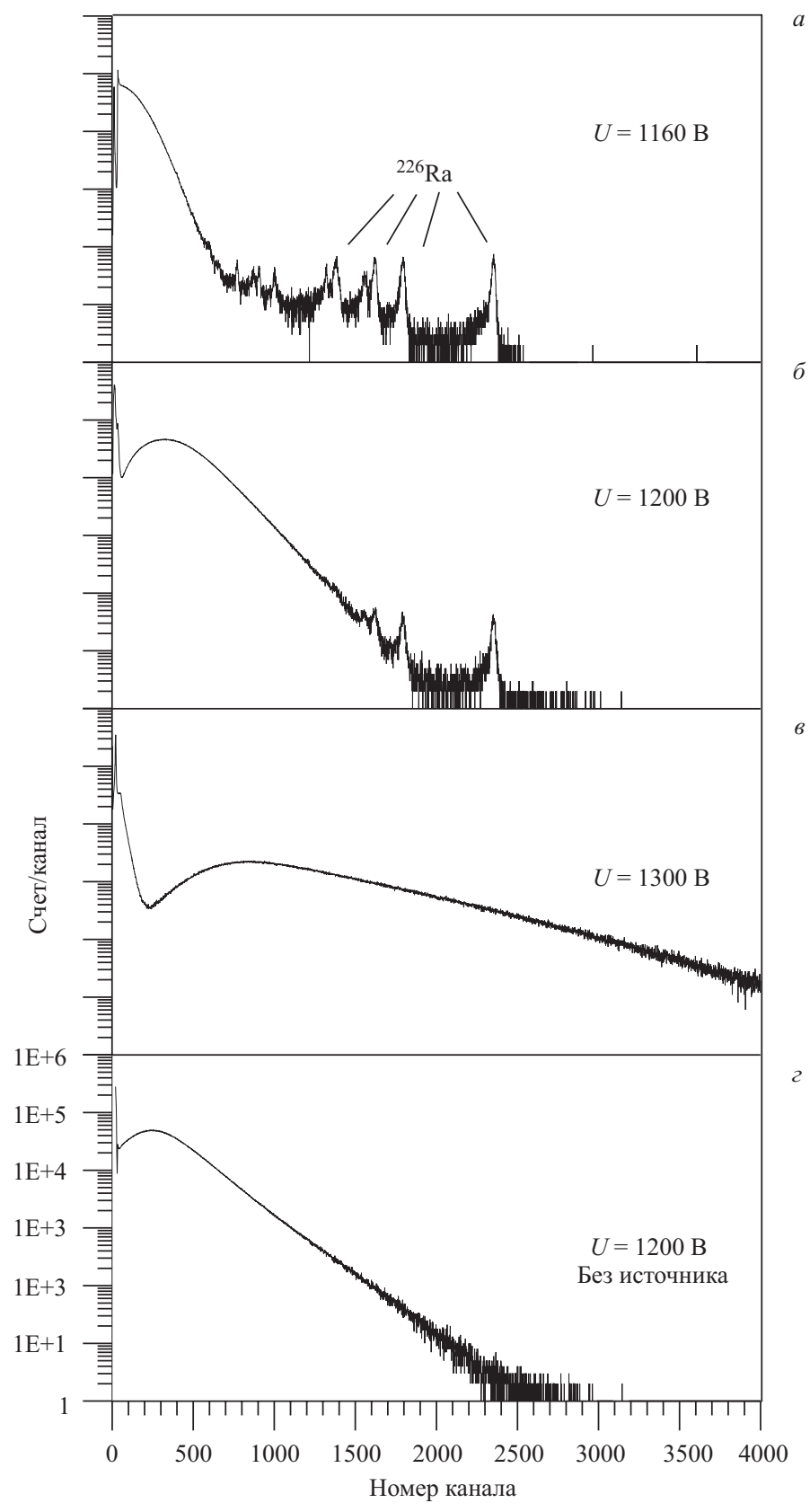

Рис. 4. Спектры, полученные с помощью стрипового детектора в режиме «генерации» с источником альфа-частиц ${ }^{226} \mathrm{Ra}(a-8)$ и без источника (2) 
регистрацией сигналов от охранных колец за счет емкостной связи кольцострип. При облучении со стороны омического контакта эти пики также присутствуют (рис. 3, в, г), но появляются при более высоких напряжениях смещения и имеют меньшую интенсивность, так как вероятность собирания носителей заряда на сигнальный электрод в этом случае значительно выше, чем на охранные кольца. Значение энергетического разрешения детекторов $\mathrm{FWHM}=60$ кэВ $(0,8 \%)$ для $E_{\alpha}=7,7 \mathrm{MэB} \mathrm{в} \mathrm{обоих} \mathrm{случаях.}$

У большинства детекторов при достижении значения напряжения $1150 \div 1200$ В появлялись сигналы, имеющие непрерывный спектр амплитуд и высокую интенсивность (см. рис. $4 a, \sigma)$. В дальнейшем будем называть этот эффект «генерацией». При повышении напряжения эффект усиливался и величины амплитуд этих сигналов превышали динамический диапазон спектрометрического тракта, а интенсивность импульсов не позволяла различить сигнал от альфа-частиц (рис. 4, в). Снижение напряжения приводило к полному восстановлению первоначальных характеристик детектора. «Генерация» наблюдалась и в отсутствие источника ионизирующего излучения (см. рис. 4, 2). Возможным объяснением «генерации» является размножение носителей зарядов (как от альфа-частиц, так и от объемных токов утечки) с переменным коэффициентом размножения, зависящим от неоднородности области сильного поля. Охлаждение детекторов до температуры жидкого азота приводило к уменьшению порога начала «генерации» до 1000 В, что связано с ростом подвижности носителей заряда по сравнению с комнатной температурой. Следует отметить, что не наблюдалось принципиальной разницы в поведении рабочих (с клиновидным профилем $p$ - $n$-перехода) и тестовых структур как при комнатной, так и при азотной температуре.

\section{ПИКСЕЛЬНЫЕ ДЕТЕКТОРЫ}

Первоначально опытные матрицы пиксельных детекторов были сделаны аналогично стриповым. В каждом квадранте располагались девять одинаковых пикселей диаметром 150 мкм, в центре которых находились клиновидные области диаметром 6 либо 15 мкм (см. рис. 5). Как и у стриповых детекторов, центральные пиксели в квадранте были тестовыми, т. е. имели стандартный профиль. Измерения этих детекторов не показали принципиальных отличий поведения их характеристик от стриповых структур. Изменилась только величина напряжения начала «генерации» ( 800 В). Это можно объяснить ростом напряженности поля (при том же напряжении смещения) из-за изменения формы и размеров $p$ - $n$-перехода. Разницы в характере поведения рабочих и тестовых структур также не было выявлено. Энергетическое разрешение для альфа-частиц составляло $\sim 0,8 \%$. 


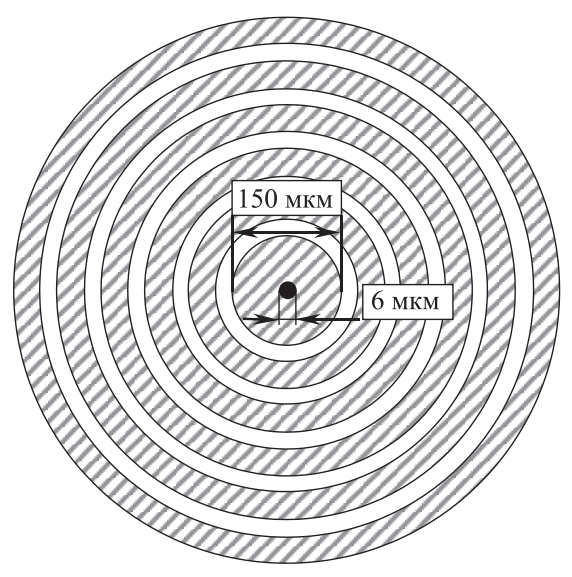

Рис. 5. Структура поверхности пиксельного детектора

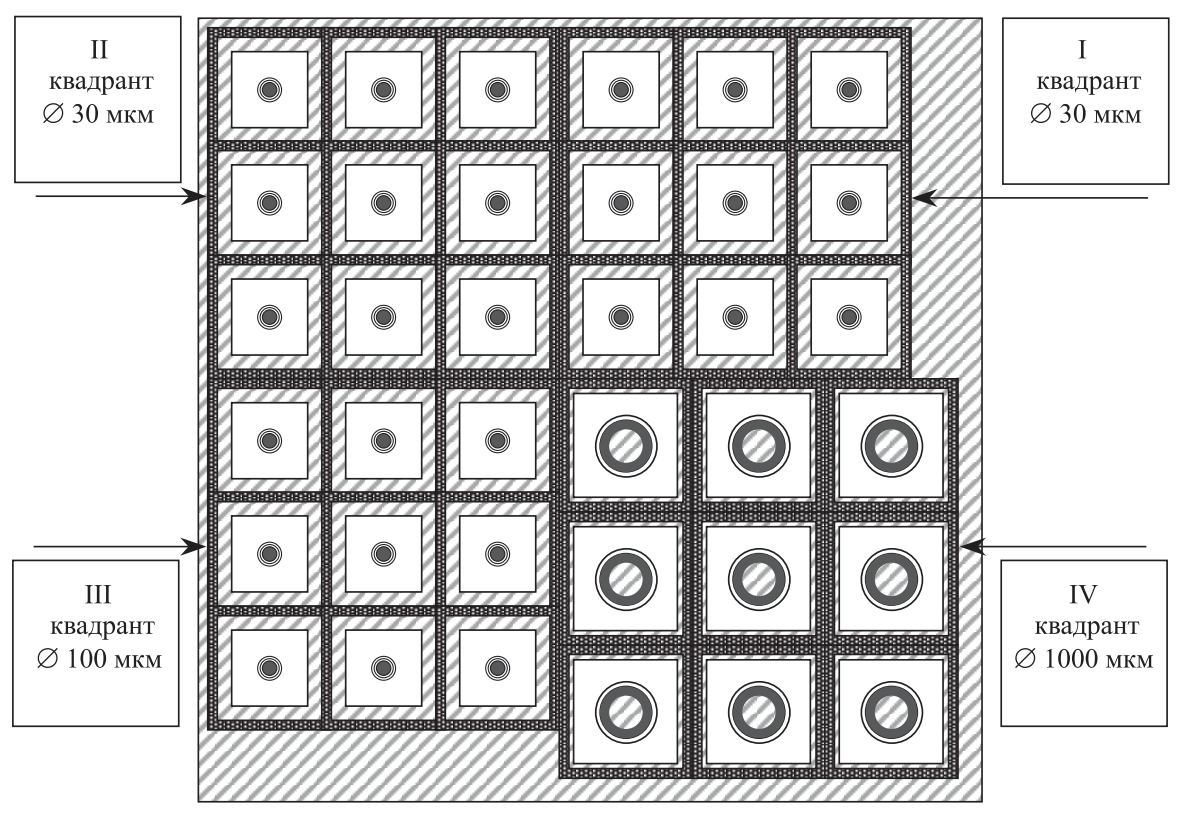

Рис. 6. Структура поверхности матрицы пиксельных детекторов 


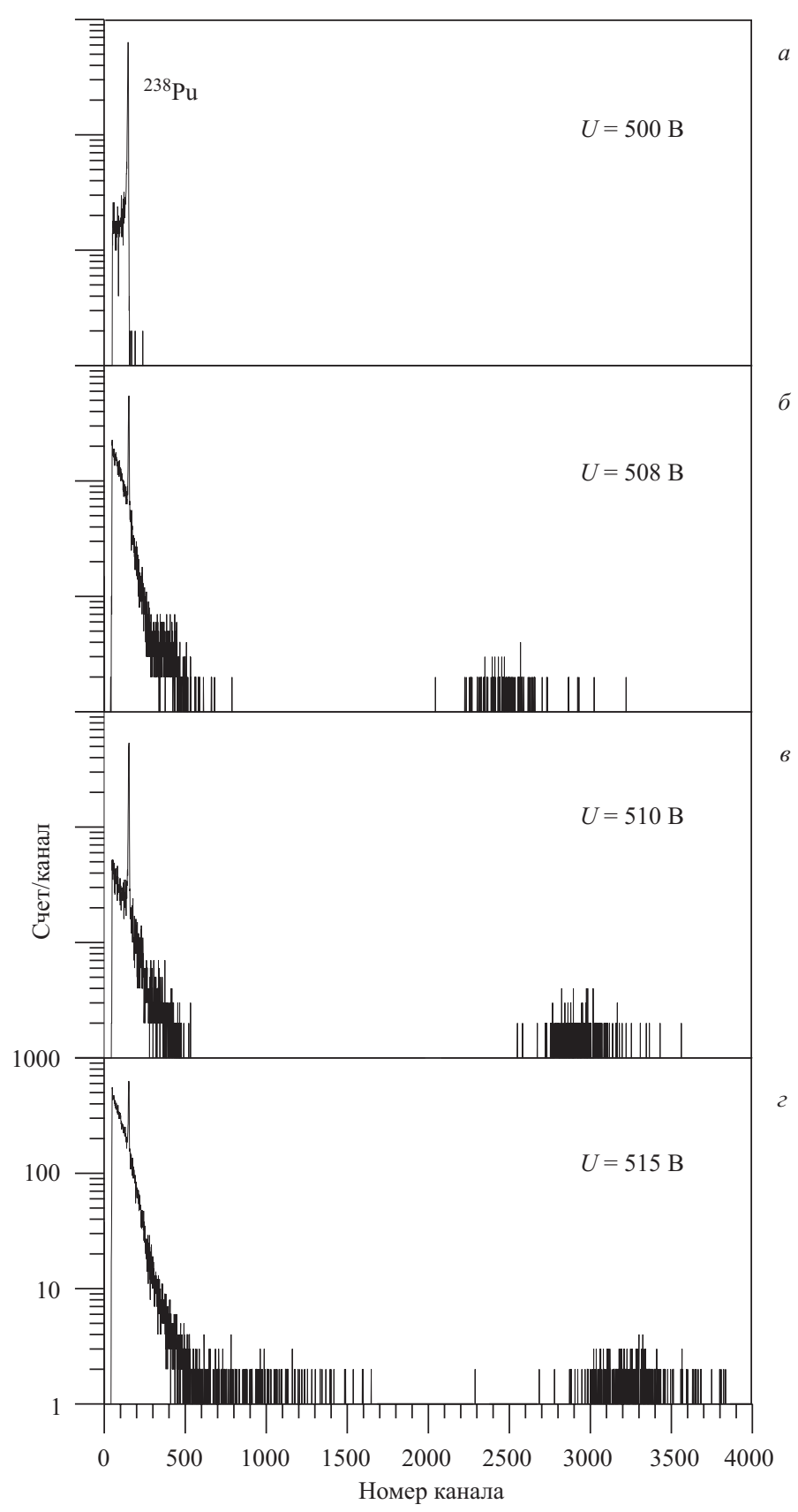

Рис. 7. Спектры альфа-частиц ${ }^{238} \mathrm{Pu}$, полученные с помощью пиксельного детектора в обычном режиме $(a)$ и в режиме усиления $(\sigma-2)$. Время измерения 30 мин 
В дальнейшем были изготовлены и исследованы девять образцов с матрицами пиксельных детекторов другого типа, отличающихся количеством и шагом охранных колец, число которых варьировалось от 2 до 12. В I и II квадрантах каждого образца располагались группы из девяти пикселей диаметром 30 мкм, в III квадранте - группы из девяти пикселей диаметром 100 мкм, а в IV - группы из девяти пикселей диаметром 1000 мкм (см. рис.6). Значение напряжения начала «генерации» для образцов, отличающихся диаметром пикселя и количеством охранных колец, изменялось в диапазоне 600-700 В.

Наиболее интересные результаты были получены при измерениях пикселя диаметром 100 мкм, имеющего два охранных кольца, при облучении со стороны омического контакта. Спектры альфа-частиц при комнатной температуре сходны со спектрами, полученными от остальных детекторов. Напряжение начала «генерации» составило 600 В. Однако при охлаждении до температуры жидкого азота (величина напряжения начала «генерации» 500 В) в спектре ${ }^{238} \mathrm{Pu}$ наряду с обычным пиком ( $E=5,5$ МэВ) появился аномальный пик с амплитудой, соответствующей значению энергии $\sim 60-70$ МэВ

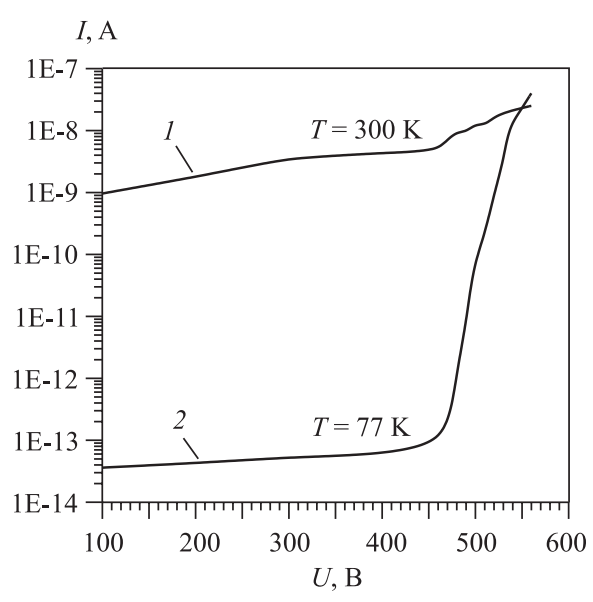

Рис. 8. Вольт-амперные зависимости пиксельного детектора при комнатной температуре (1) и температуре жидкого азота (2) (см. рис. 7). На спектрах не видны пики от охранных колец из-за того, что коэффициент усиления спектрометрического усилителя в этих измерениях был минимально возможным. Положение аномального пика зависело от величины напряжения смещения в узком диапазоне (508515 В). Кроме того, с увеличением значения напряжения в этом интервале наблюдался рост числа событий под аномальным пиком и соответствующее падение интенсивности обычного пика ${ }^{238} \mathrm{Pu}(E=$ 5,5 МэВ). Для напряжения 508 В интенсивность аномального пика составила $4 \%$ от интенсивности обычного, для 510 В - $6 \%$ и для 515 В - 8\%. При этом суммарная интенсивность обоих пиков сохранялась. Дальнейшее повышение величины напряжения на детекторе вело к значительному росту «генерации» и смещению аномального пика за предел диапазона спектрометрического тракта.

На рис. 8 приведены вольт-амперные характеристики детектора, полученные в разных температурных условиях. Резкое возрастание значений токов детектора при температуре жидкого азота в области напряжений, где по- 


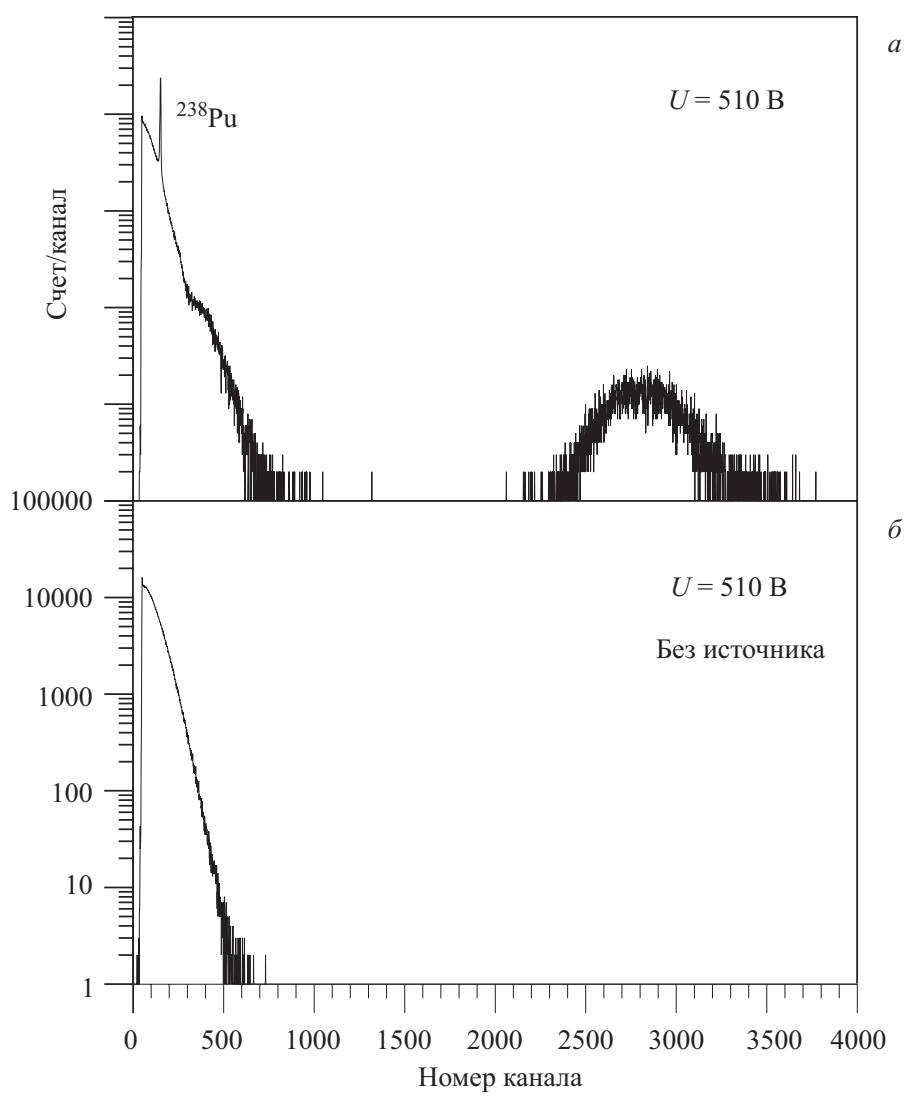

Рис. 9. Спектры, полученные с помощью пиксельного детектора в режиме усиления с источником альфа-частиц ${ }^{238} \mathrm{Pu}(a)$ и без источника (б). Время измерения 24 часа

является «генерация» и аномальный пик, также указывает на существование в данном образце режима внутреннего усиления и связь эффекта «генерации» с мультипликацией носителей заряда. В то же время надо отметить, что наблюдаемые вместе с усиленными пиками обычные пики от альфа-частиц говорят о том, что лишь небольшая часть носителей заряда попадает в область мультипликации. Спектр альфа-частиц ${ }^{238} \mathrm{Pu}$, полученный за 24 часа измерений (рис. 9), демонстрирует, что усиленный пик имеет следующие параметры: положение пика соответствует значению энергии 70,2 МэВ, энергетическое разрешение FWHM = 10,2 МэВ. Результаты, полученные при облучении детектора альфа-частицами ${ }^{226} \mathrm{Ra}(E=4,8,5,5,6,0$ и 7,7 МэВ), показали, что усиленные пики явно не разделяются. Это не позволило нам установить связь коэффициента внутреннего усиления с величиной энергии частиц. 


\section{ЗАКЛЮЧЕНИЕ}

Полученные результаты указывают на возможность реализации в планарном кремниевом детекторе режима внутреннего усиления, сходного с пропорциональным усилением в газовых счетчиках. На наш взгляд, это может быть первым шагом в разработке более массивных детекторов подобного типа, в частности на основе HPGe.

Другим немаловажным фактом является то, что исследованные детекторы (работая в обычном режиме) показали высокие спектрометрические характеристики при большом перенапряжении (приблизительно в 10 раз превышающем напряжение обеднения). Это позволяет использовать их в ряде экспериментов, где требуются хорошие временные свойства, например, в качестве быстрых триггеров.

Авторы выражают благодарность Л.Н.Богдановой и В.Б.Бруданину за постоянное внимание к работе. Исследование выполнено при поддержке Департамента атомной науки и техники Министерства Российской Федерации по атомной энергии, государственные контракты 66.04.19.19.00.802, 6.04.19.19.01.908, 6.25.19.19.02.969, и частично поддержана Американским фондом гражданских исследований и развития, гранты RP1-2111, RP2-2383.

\section{ЛИТЕРАТУРА}

1. Morales A. // Nucl. Phys. Proc. Suppl. 2002. V. 110. P. 39;

Bergstrom L. // Rep. Prog. Phys. 2000. V.63. P.793

2. Беда А. Г. и др. // ЯФ. 1998. Т. 61, № 1. С. 66;

Неганов Б. С., Трофимов В. Н., Юхимчук А. А. // ЯФ. 2001. Т. 64, № 11. С. 1948.

3. Cabrera B., Krauss L. M., Wilczek M. // Phys. Rev. Lett. 1985. V.55. P. 25.

4. De Bellefon A. et al. // Astropart. Phys. 1996. V.6. P.35;

Benoit A. et al. // Phys. Lett. B. 2002. V.545. P. 43.

5. Huth G. C. // Proc. Gatlinburg Conference. 1969. P. 323.

6. Moszynski M. et al. // IEEE Trans. Nucl. Sci. 2001. V. 48, № 1. P. 1205.

7. Shah K. S. et al. // Ibid. №6. P. 2352.

8. Madden T. C., Miller G. L. // Proc. Gatlinburg Conference. 1969. P. 314.

9. Lutz G. // Nucl. Instrum. Meth. Phys. Res. 2003. V. A501. P. 288.

10. Ripamonti G. et al. // Nucl. Instrum. Meth. Phys. Res. 1990. V. A288. P. 99. 
11. Бурбаев T.M. $u$ др. // Краткие сообщения по физике ФИАН. 1992. № 3 , 4. C. 34 .

12. Беда А. Г., Старостин А. С. // ЯФ. 2000. Т. 63, № 7. С. 1370.

13. Bloch P. et al. // IEEE Trans. Nucl. Sci. 2002. V.49. P. 321.

14. Goulding F. S. // Nucl. Instrum. Meth. 1966. V.43. P. 1.

\author{
Получено 3 марта 2004 г.
}




\section{Редактор О. Г. Андреева \\ Макет E. В. Сабаевой}

Подписано в печать 27.04.2004.

Формат $60 \times 90 / 16$. Бумага офсетная. Печать офсетная.

Усл. печ. л. 0,94. Уч.-изд. л. 1,14. Тираж 320 экз. Заказ № 54408.

Издательский отдел Объединенного института ядерных исследований 141980, г. Дубна, Московская обл., ул. Жолио-Кюри, 6.

E-mail: publish@pds.jinr.ru www.jinr.ru/publish/ 